\title{
COM OS PÉS NUM MUNDO NOVO: SAÚDE INDÍGENA NO DISTRITO SANITÁRIO ESPECIAL DO TOCANTINS
}

\section{WITH FEET IN A NEW WORLD: INDIGENOUS HEALTH IN THE SPECIAL SANITARY DISTRICT OF TOCANTINS}

Rafael Souza Silva ${ }^{1}$, Heide Souza Porto ${ }^{2}$, Thaís Caire da Silva Costa ${ }^{3}$, Leidiany Souza Silva ${ }^{4}$, Rogério Carvalho de Figueredo ${ }^{5}$

\section{RESUMO}

Introdução: A atuação da enfermagem nas áreas indígenas é de fundamental importância tendo em vista, a dificuldade em que estes têm desde o deslocamento para os locais de saúde até fatores culturais complexos que impedem de sair da aldeia. Objetivo: descrever a atuação do enfermeiro no atendimento de saúde dos Povos Indígenas do Distrito Sanitário Especial Indígena do Tocantins (DSEI-TO). Material e Métodos: trata-se de uma pesquisa de campo de caráter retrospectivo, descritivo e explorativa com análise documental. $O$ estudo foi realizado através de avaliação de relatórios públicos documentais e eletrônicos produzidos pelo DSEI-TO. Resultados e Discussão: A partir da análise dos documentos foi possível caracterizar o papel do DSEI, bem como a realidade da assistência a saúde prestada à população indígena. Com base nos dados, observa-se uma evolução nos cuidados durante o pré-natal, taxa de mortalidade infantil, saúde bucal, estado de imunização conforme o Programa Nacional de Imunização nas diferentes fases do ciclo vital. Considerações Finais: a assistência à saúde prestada a população indígena, principalmente pela enfermagem, é baseada em improvisos que podem ter sentidos opostos, ou seja: positivo, pela superação das dificuldades enfrentadas, e negativo, por colocar em prática uma atuação limitada e com incerteza da efetividade.

Palavras-chave: Enfermeiro, atenção primaria, Saúde Indígena.

\section{ABSTRACT}

Introduction: The role of nursing in indigenous areas is of fundamental importance in view of the difficulty they face, from moving to health facilities to complex cultural factors that prevent them from leaving the village. Objective: to describe the role of nurses in health care for Indigenous Peoples in the Special Indigenous Health District of Tocantins (DSEI-TO). Material and Methods: this is a retrospective, descriptive and exploratory field research with documentary analysis. The study was carried out through the evaluation of documentary and electronic public reports produced by DSEITO. Results and Discussion: From the analysis of the documents, it was possible to characterize the role of the DSEI, as well as the reality of health care provided to the indigenous population. Based on the data, there is an evolution in care during prenatal care, infant mortality rate, oral health, immunization status according to the National Immunization Program at different stages of the life cycle. Final Considerations: the health care provided to the indigenous population, mainly by nursing, is based on improvisations that may have opposite meanings, that is: positive, by overcoming the difficulties faced, and negative, by putting in practice a limited performance and with uncertainty effectiveness.

Keywords: Nurses, Primary Care, Indigenous Health.

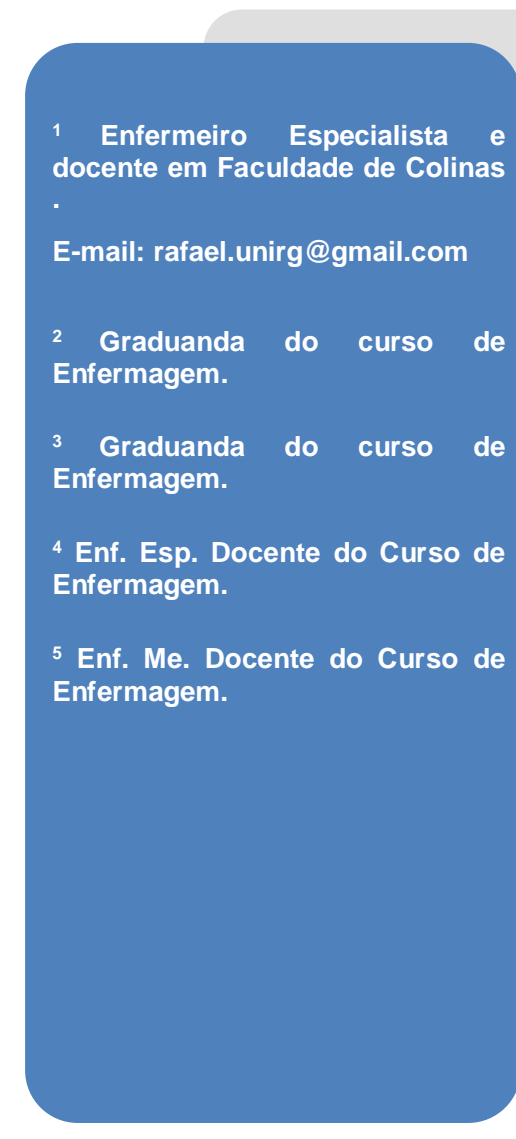




\section{INTRODUÇÄOO}

A saúde indígena tem sido entendida como um dos campos de atuação voltada especialmente a saúde coletiva, tendo como uma das principais características a interculturalidade. Nesse contexto, toda a prática dos profissionais da saúde é direcionada a população indígena que é na verdade o centro intercultural, um espaço aonde há o encontro de dois sistemas diferentes mas que atuam com o mesmo proposito a cura, com visões e práticas diferenciadas que são levados ao diálogo, para então, chegar a um senso comum.

De um lado encontra-se o médico ocidental que se baseia em condutas de saberes presentes na racionalidade científica. Do outro, os sistemas indígenas de saúde com suas práticas de cuidado e cura, que vem dos saberes repassados dos seus antepassados, que utilizava a natureza, misticidade para obter a cura. (NOVO, 2011)

Neste espaço de interlocução, o que se faz presente é a coexistência de duas sociedades, que consequentemente possui a presença de dois sistemas médicos diferentes e em uma discrepante desigualdade em relação ao poder. (MENDONÇA, 2015)

O processo de redução de adoecimento em sua ampla dimensão, no qual envolve tanto a biologia quanto a individualidade do ser, é um traço que evidencia o modelo médico empregado na sociedade e que acaba se contrapondo a todos os paradigmas que abrangem a população indígena, onde se percebe seu aspecto sociocultural.

Nas concepções do povo indígena, as doenças atingem não apenas a matéria "corpo", mas tem uma relação pessoal com a sociedade e isto, gera implicações e impactos de maneira coletiva exercendo as pessoais, fazendo com que a biomedicina não seja capaz de comprovar todos os eventos que ocorrem na vida de um ser humano, havendo "coisas" que somente a natureza tem a resposta (BUCHILET, 2006).

Assim, o enfermeiro, encontra-se em uma posição conflituosa, pois ao mesmo tempo que é responsável pela promoção e agente do modelo biomédico aplicado na sociedade, vivencia as dificuldades de implementar condutas de saúde dentro da cultura indígena, que se recusa a aceitar a mudanças. Durante todo o convívio com esse público, o profissional é levado a refletir acerca dos seus valores éticos e morais, saberes e práticas.

De acordo com Fernandes (2010) a decisão de trabalhar na saúde indígena acaba gerando no profissional a necessidade de reflexão acerca da cultura que permeou sua existência. 
Nessa ótica, Enfermeiros deverão estar preparados para trabalhar em meio essa diversidade, buscando sempre a melhor forma de levar a assistência, fazendo uso dos conhecimentos em gerenciamento, assistencialismo, planejamento... com intuito de ofertar uma assistência humanizada, sendo capaz de avaliar recursos tecnológicos, organizacionais e humanos exigidos para a criação e a gestão do conhecimento, desenvolvendo dessa maneira, competências, habilidades, atitudes e valores para planejar, organizar, dirigir e controlar a gestão do conhecimento nas organizações de seu capital humano.

No estado Tocantins a assistência à saúde aos Povos Indígenas encontra-se sob a responsabilidade sanitária do Distrito Sanitário Especial de Saúde Indígena do Tocantins (DSEI/TO), sendo a SEDE instalada na capital do estado Palmas, possuindo uma área habitada por 11(onze) grupos étnicos de diferentes culturas, todos possuem relação social com a sociedade não indígena, totalizando uma população de 11.199 (onze mil, cento e noventa e nove) indígenas, distribuídas em 190 (cento e noventa) aldeias, das quais apenas 162 (cento e sessenta e duas) estão inseridas no Sistema de Informação da Atenção à Saúde Indígena (SIASI), com 1.085 (mil e oitenta e cinco) citadinos residentes nos municípios de Palmas, Araguaína e Gurupi. (DSEI, 2017).

Diante, da grande relevância em saúde indígena, o enfermeiro é o profissional que ao mesmo tempo em que é um representante fidedigno da cultura do seu povo, é também posto como um porta da voz do povo indígena, sendo desse modo indispensável desde da assistência básica até a mais complexa, o que justifica-se a importância de conhecer seu campo de atuação dentro das comunidades. De modo, que seja levado em consideração todo o contexto que envolve o trabalho com essa etnia.

Diante da necessidade de detalhar a atuação do enfermeiro no atendimento à população indígena, tem-se como objetivo descrever as responsabilidades do enfermeiro, na atenção primária em saúde das comunidades indígenas presentes no Estado do Tocantins, abordando sobre as principais dificuldades desses profissionais na inserção de um modelo biomédico ocidental, além das barreiras existentes pela cultura.

Nesse sentido, proporcionar a ampliação do conhecimento se torna um combustível para o avanço do trabalho, e levando em consideração que grande parte dos profissionais que estarão integrando este serviço não conhecem a real necessidade do público, discutir este papel é um avanço tanto para a profissão quanto para uma maior satisfação da população indígena. 


\section{MATERIAIS E METODOS}

O método aplicado foi pesquisa de campo de caráter retrospectivo, descritivo e explorativa com análise documental. O estudo foi realizado através de avaliação de relatórios públicos documentais e eletrônicos produzidos pelo DSEI-TO durante o ano de 2018.

De acordo com a resolução 466/12 do Conselho Nacional de Saúde, que define as diretrizes e normas reguladoras das pesquisas envolvendo seres humanos, o presente estudo não foi submetido à aprovação ao Comitê de Ética por se tratar de pesquisa em relatórios públicos.

Considerando a base do estudo, o critério de inclusão foi relatórios públicos que continham informações sobre os indicadores da saúde indígena na atenção primária. Foram selecionados os relatórios publicados que continha no máximo 5 anos de sua publicação e que abrangiam com ênfase as políticas públicas realizadas à população indígena na atenção primária em saúde. Para tal utilizamos relatórios de 2015 a 2018, totalizando 8 relatórios explorados no presente trabalho.

Foram excluídos da base de pesquisa os relatórios produzidos que não continham informações sobre indicadores da saúde indígena na atenção primária em saúde e que foram publicados há mais de 5 anos.

A pesquisa foi realizada durante o mês de julho de 2019, na Coordenação do DSEITO em Palmas - TO. Foram levantados 12 (doze) relatórios públicos, sendo que 08 (oito) atendiam os critérios de inclusão e 04 (quatro) foram excluídos.

Os riscos relacionados a presente pesquisa envolvem cuidados na análise, devido a vulnerabilidade do público mencionado. Para que não ocorra a evasão de informação a pesquisa será realizada em ambiente privativo. O benefício envolvido está relacionado ao aprimoramento da capacidade dos profissionais de enfermagem, em relação ao trabalho do Enfermeiro na Saúde Indígena.

Os resultados foram produzidos com base na avaliação dos relatórios e foram apresentados através de tabelas, gráficos com suas respectivas discussões teóricas.

\section{RESULTADOS E DISCUSSOES}

Em outubro de 2010, a partir da criação da Secretaria Especial de Saúde Indígena (SESAI/MS), através do Decreto Presidencial no 7.336 de 20/10/2010, as ações de 
Atenção a Saúde Indígena, até então sob a responsabilidade da Fundação Nacional de Saúde (FUNASA), passaram a ser de responsabilidade do(s) Distrito(s) Sanitário(s) Especial Indígena (DSEls), que foram criados pela Portaria ํo 852 de 30 de setembro de 1999, da Fundação Nacional de Saúde (FUNASA), com a competência de atuar como unidade de execução das ações destinadas a promoção, proteção e recuperação à saúde do Índio.

O Ministério da Saúde considera que, a assistência e a promoção da saúde nas comunidades indígenas apresentam impacto significativo nas condições de saúde e qualidade de vida dessa população. O Enfermeiro no âmbito da saúde dos povos Indígenas atua completando a Equipe Multidisciplinar de saúde Indígena (EMSI) que e composta por Enfermeiros, Médicos, odontólogos, técnico em enfermagem, técnico em saúde bucal, agente Indígena de saúde (AIS) agente Indígena de Saneamento (AISAN), segundo a Portaria ํㅜ 1.088, de 04 de Julho de 2005 fica garantida a possibilidade de inserção nas equipes multidisciplinares de saúde indígena de outros profissionais que atuam na saúde indígena, de acordo com a situação epidemiológica e necessidades de saúde.

Corroborando com a Política Nacional de Atenção Básica (PNAB) a Política Nacional de Atenção à Saúde dos Povos Indígenas (PNASPI) esperar resolver $80 \%$ dos agravos à saúde, que deverão ser resolvidas nesse nível, desta forma a organização da assistência à saúde para cada Distrito Sanitário Especial Indígena (DSEI), se dá a partir da definição de uma rede de serviços de atenção básica de saúde dentro das áreas indígenas, integrada e hierarquizada com complexidade crescente e articulada com a rede do SUS. Nesta organização dos serviços de saúde, as comunidades têm uma outra instância de atendimento, que são os Polos-Base (PB), a primeira referência para os Agentes Indígenas de Saúde (AIS) que atuam nas aldeias, nas aldeias, a atenção básica será realizada por intermédio dos Agentes Indígenas de Saúde, nos postos de saúde, e pelas equipes multidisciplinares periodicamente, conforme planejamento das suas ações. (BRASIL, 2002).

Com objetivo de garantir a integralidade da assistência dos Povos Indígenas, a Lei no 9.836, de 23 de setembro de 1999, atrás em seu Art. 19 G nos incisos $2^{\circ}$ e 3oㅡ respectivamente:

§ 20 O SUS servirá de retaguarda e referência ao Subsistema de Atenção à Saúde Indígena, devendo, para isso, ocorrer adaptações na estrutura e organização do SUS nas regiões onde residem as populações indígenas, para propiciar essa integração 
e o atendimento necessário em todos os níveis, sem discriminações.

§ 30 As populações indígenas devem ter acesso garantido ao SUS, em âmbito local, regional e de centros especializados, de acordo com suas necessidades, compreendendo a atenção primária, secundária e terciária à saúde.

Atendo ao disposto na (PNASPI), as demandas não atendidas no grau de resolutividade dos Polos Base (PBs) devem ser referenciadas para a rede de serviços do SUS e contam com apoio da Casa de Saúde Indígena (CASAI), que é um estabelecimento responsável pelo apoio, acolhimento e assistência aos indígenas referenciados aos demais serviços do SUS, para realização de ações complementares de atenção básica e de atenção especializada, sendo também destinada a seus acompanhantes, quando necessário. (BRASIL, 2002).

$\mathrm{Na}$ produção do cuidado ao indígena, os profissionais de enfermagem se deparam diariamente com situações percepções distintas, o profissional enfermeiro é responsável pela gestão das ações de saúde realizadas nos Polos Bases e tem ainda o papel estratégico na organização dos serviços de saúde do seu território.

De acordo com as Notas Técnica 17/2018 e 018/2014 do Departamento de Atenção á Saúde Indígena (DASI) da Secretaria Especial de Saúde Indígena (SESAI/MS), o profissional enfermeiro desenvolver vários funções na execução e fortalecimento da atenção básica para os Povos Indígenas, desde da gestão até a assistência na produção do cuidado, desempenhando vários papéis tais, como:

$\checkmark \quad$ Planejar, Monitorar e Avaliar as ações de saúde e situações de riscos;

$\checkmark \quad$ Construir o planejamento baseado na análise da Situação de Saúde dos Territórios (foco na vigilância);

$\checkmark \quad$ Planejar atividades de Educação Permanente;

$\checkmark \quad$ Planejar ações de Educação em Saúde, Subsidiar e apoiar a elaboração da Programação das atividades Junto às EMSI;

$\checkmark \quad$ Planejar as ações de prevenção de doenças e de promoção da saúde, assim como estratégias de cuidado a serem realizadas no domicílio pelas EMSI;

Apoiar a CASAI no planejamento das Ações de Saúde e acompanhar a produção da Equipe de Saúde das Casai;

$\checkmark$ Acolher os usuários, proporcionando atendimento humanizado, responsabilizando-se pela continuidade da atenção e viabilizando o estabelecimento do vínculo; 
$\checkmark \quad$ Participar das reuniões das instâncias colegiadas (conselhos municipais e/ou estaduais de saúde, comissões locais Comissão Intergestores Regional (CIR), Comissão Intergestores Bipartite (CIB) e outros espaços interinstitucionais;

$\checkmark \quad$ Organizar o serviço de forma que a notificação no Sistema de Informações de Atenção à Saúde Indígena (SIASI) e demais sistemas do SUS sejam parte da rotina de trabalho;

$\checkmark \quad$ Garantir o registro de dados no SIASI e demais sistemas do SUS de forma oportuna e qualificada;

$\checkmark \quad$ Auxiliar as EMSI na elaboração da cartografia social de seus territórios;

$\checkmark \quad$ Fomentar a realização de espaços de escuta e troca de conhecimentos com os indígenas, reconhecendo o protagonismo deles no cuidado à saúde e valorizando os saberes, práticas e especialistas tradicionais;

$\checkmark \quad$ Articular a realização e participar de reuniões técnicas e capacitações organizadas pelos municípios e/ou estados, quando couber e/ou houver afinidade temática, oportunizar vagas aos municípios e estado quando houver capacitações que podem fortalecer as Redes de Atenção à Saúde (RAS) entre outros.

Figura 1. Organização dos DSEl'S e Modelo Assistencial.

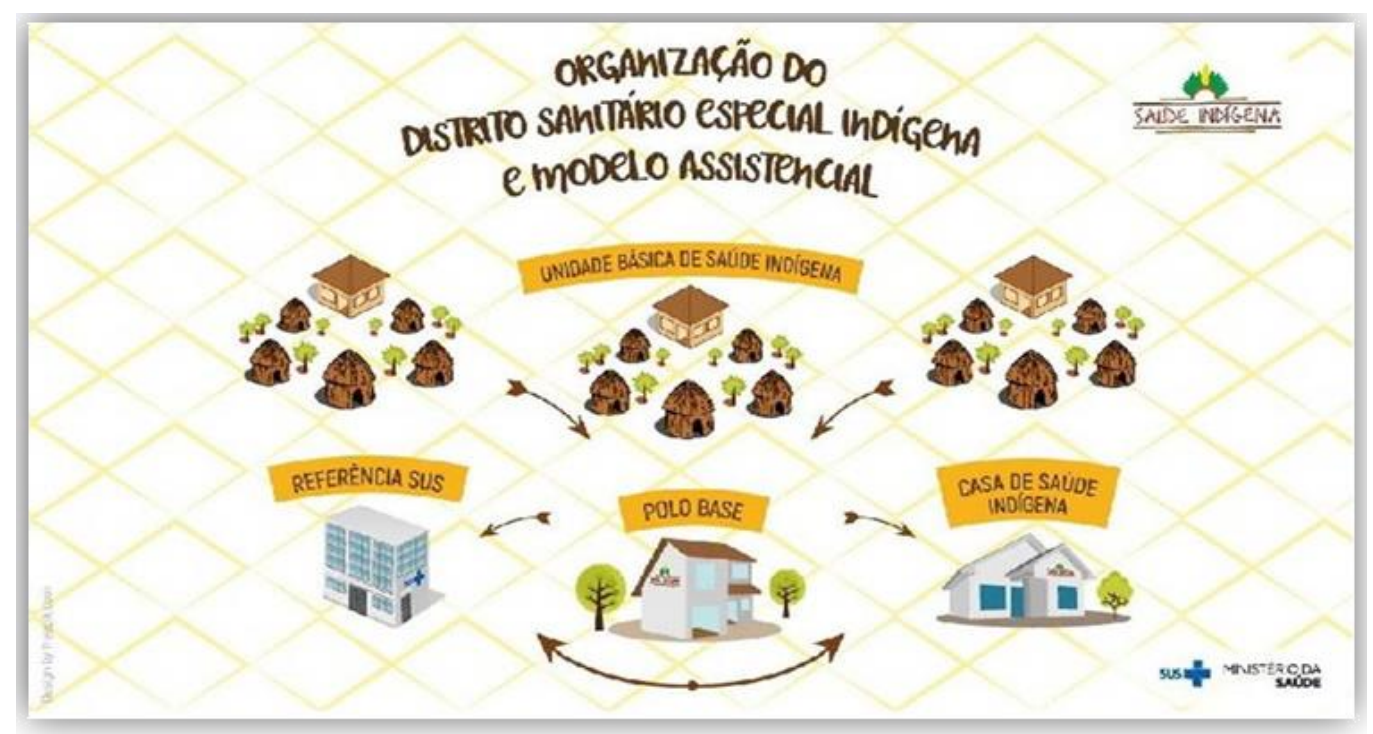

Fonte: http://portalsaude.saude.gov.br/2018.

O Distrito Sanitário Especial Indígena do Tocantins (DSEI- TO) tem abrangência em toda a extensão do Estado do Tocantins, em 15 (quinze) municípios com terras e população indígena residentes, a saber: Araguaína (Citadinos); Araguaçu; Formoso do Araguaia; Goiatins; Gurupi (Citadinos); Itacajá; Lagoa da Confusão; Maurilândia do 
Tocantins; Pedro Afonso; Sandolândia; Santa Fé do Araguaia; Tocantinópolis; Palmas (Citadinos); Cachoeirinha, São Bento, Pium e no Sul do Estado do Pará no município de Santa Maria das Barreiras.

O DSEI-Tocantins tem atualmente a população de aproximadamente 12.136 (doze mil, cento e trinta e seis) indígenas aldeados e aproximadamente 202 (duzentos e doze) citadinos que são indígenas que vivem em contexto urban. Os indígenas aldeados que são de responsabilidade sanitária para atenção básica do DSEI - TO, estão distribuídos em 172 (cento e setenta e duas ) aldeias, divididas em 05 (cinco) Polo Base Indígena (PBI) nos estados de Tocantins e Pará, representados por 11 grupos étnicos: etnia Apinajé, Funi-ô, Krahô, Karajá-Xambioá e Santana, Xerente, Javaé, Karajá, Kanela, Pankararu, Atikum, dentre outras etnias; todos possuem contato com a sociedade não indígena.

O acesso às Terras e Territórios Indígenas ocorre pelos modos terrestre e fluvial, em uma extensão territorial de 2.202.776 (dois milhões duzentos e dois mil setecentos e setenta e seis hectares) $\mathrm{km}^{2}$ (DSEI/TO, 2017).

O DSEI /TO possui 05 (cinco) Polos Base Indígena, 04 (quatro) Bases de Apoio, 02 (duas) Casa de Saúde Indígena (CASAI) e 30 (trinta) Unidades Básicas de Saúde Indígena (UBSI), nos quais atuam 13 (treze) Equipes Multidisciplinares de Saúde Indígena (EMSI) compostas pelos seguintes profissionais: médico, cirurgião-dentista, enfermeiros, técnicos de enfermagem, técnico em saúde bucal, agente indígena de saúde (AIS) e agente indígena de saneamento (AISAN), sendo 09 EMSI completas e 04 incompletas (sem médico e cirurgião-dentista), garantindo assim 100\% de cobertura assistencial para a população atendida pelo distrito, as EMSI se deslocam de $2^{\underline{a}}$ a $6^{\underline{a}}$ feira para os territórios e as demandas que não atendidas da Atenção Básica, são encaminhadas para os municípios de referência para Média e Alta complexidade, conforme previsto na Lei no 9.836, de 23 de setembro de 1999, (DSEI/TO, 2017).

Ao DSEI, cabe ainda garantir o suporte para a assistência de média e alta complexidade e para tal é imprescindível que este disponha de uma rede de atenção para os serviços de atenção primária sendo organizado hierarquicamente de forma que seja possível a articulação com toda a rede de serviços do Sistema Único de Saúde (SUS). (BRASIL, 2016).

Interessante salientar que o Decreto no: 3.156/99, da criação do SASI SUS, mudou o modelo de oferta de serviços de saúde até então prestados aos indígenas, que era focado somente no suporte emergencial ou através de convênio para assistência a saúde 
mantida sob a responsabilidade da FUNAI, tratava-se de um modelo com ações imediatistas voltadas ao controle epidêmico, deixando de lado ações de promoção e prevenção em saúde para esses povos. Na incorporação da Saúde Indígena pelo MS, com gestão do SUS, foi adotado novo modelo assistencial, focado nas questões culturais e a premissa de um subsistema de saúde que respeitasse as crenças e anseios desses povos, configurados agora no formato de Distrito Sanitário Especial Indígena (DSEI).

Como dito acima, um Distrito Sanitário Especial Indígena (DSEI) tem como atribuição, observar as áreas territoriais indígenas de sua responsabilidade, bem como a demanda de infraestrutura e de pessoal que seja necessário para a execução efetiva dos serviços de atenção a saúde às comunidades indígenas residentes.

No que se refere às necessidades de profissionais de saúde para atuar em territórios diferenciados culturalmente foram criadas as Equipes Multidisciplinares de Saúde Indígena (EMSI), para prestação de serviços nas Unidades Básicas de Saúde Indígena (UBSI).

Também foi implantado o principal programa estratégico do SASI, que foi a capacitação de pessoas indígenas, nativas e residentes nas aldeias, para trabalharem diretamente em suas comunidades como Agentes Indígenas de Saúde (AIS) e Agentes Indígenas de Saneamento (AISAN). (LANGDON e CARDOSO, 2015).

A Portaria nº 475/2008, determina que uma Equipe Multidisciplinar de Saúde Indígena (EMSI), deve ser composta basicamente pelos seguintes profissionais: médico clínico, enfermeiro, técnico de enfermagem, odontólogo (clínico ou cirurgião), técnico ou auxiliar em saúde bucal, agente indígena de saneamento (AISAN), técnico de saneamento e agente indígena de saúde (AIS). (BRASIL, 2008).

Por meio da Portaria GM no $1801 / 2015$ os postos de saúde se tornaram Unidades Básicas de Saúde Indígena (UBSI), definidos como uma unidade de saúde que deve ficar localizada em aldeia indígena, voltada para a atenção direta de assistência à saúde e saneamento. Deve também dispor de uma estruturação bem definida e adequada de acordo com as necessidade e particularidades das comunidades indígenas que são assistidas pelos serviços de saúde. (AMORIM, 2016).

A referida portaria acima define também Polos Bases, que são considerados departamentos dos DSEI e o suporte necessário para que EMSI's planejem de forma técnica e administrativa os serviços de atenção a saúde que serão ofertados para a população indígena adstrita; sendo também considerado a primeira referência para AISs e 
AISAN, que atuam diretamente em suas aldeias; sua sede pode ainda ser edificada na própria aldeia ou em municípios próximos da mesma. (BRASIL, 2015).

A tabela 1 apresenta a distribuição populacional das Comunidades Indígenas no DSEI/TO por município de abrangência, etnias, número de aldeias, número de Unidades Básicas de Saúde Indígena (UBSI), Casa de Saúde Indígena (CASAI) e Polo Base Indígena (PBI) de referência em 2018, demonstrando o contexto de apoio de serviços de saúde do DSEI/TO.

Tabela 1: Distribuição da População Indígena do DSEI Tocantins, por Município, Etnia, Aldeia, UBSIS e Polo Base, no ano de 2018.

\begin{tabular}{|c|c|c|c|c|c|}
\hline $\begin{array}{l}\text { Município de } \\
\text { Abrangência }\end{array}$ & Etnias & População & Aldeia & UBSI & $\begin{array}{l}\text { Polo Base } \\
\text { Indígena }\end{array}$ \\
\hline Formoso do Araguaia & Javaé- Karajá & 1.767 & 18 & 06 & Polo Base \\
\hline Lagoa da Confusão & da Ilha- Krahô & & & & Indígena de \\
\hline Araguaçu & Kanela, Krahô & & & & Formoso do \\
\hline Sandolândia & e Avá- & & & & Araguaia \\
\hline Pium & Canoeiro & & & & \\
\hline Itacajá & Krahô & 3.591 & 38 & 10 & Polo Base \\
\hline Goiatins & & & & & $\begin{array}{l}\text { Indígena de } \\
\text { Itacajá }\end{array}$ \\
\hline Santa Fé do Araguaia & Karajá - & 400 & 06 & 03 & Polo Base \\
\hline Santa Maria das & Xambioá e & & & & Indígena de \\
\hline Barreiras (PA) & Santana & & & & $\begin{array}{c}\text { Santa Fé do } \\
\text { Araguaia }\end{array}$ \\
\hline Tocantínia & Xerente & 3.705 & 68 & 06 & Polo Base \\
\hline Pedro Afonso & & & & & $\begin{array}{l}\text { Indígena de } \\
\text { Tocantínia }\end{array}$ \\
\hline Tocantinópolis & Apinajé e & 2.754 & 42 & 05 & Polo Base \\
\hline Maurilândia & Fulni-ô & & & & Indígena de \\
\hline Cachoeirinha & & & & & Tocantinópolis \\
\hline São Bento & & & & & \\
\hline
\end{tabular}

Casa de Saúde Indígena

de Araguaína CASAIS

Casa de Saúde Indígena 


\section{de Gurupi}

\section{Setor de Enfermagem de}

Palmas

\section{Municípios (DSEI/TO)}

12.136

172

30

Fonte: SIASI/DSEI-TO, 2018.

A Política Nacional de Atenção à Saúde dos Povos Indígenas (PNASI) garante aos indígenas aldeados que todas as ações de atenção básica sejam realizadas na terra indígena e de acordo com os dados do Sistema de Informação da Saúde Indígena (SIASI), os indicadores de saúde do DSEI - TO nos últimos 04 anos reflete 0 fortalecimento da Atenção Primária.

Gráfico 1: Percentual de partos realizados em indígenas com 06 ou mais consultas de pré-natal - 2015 a 2017.

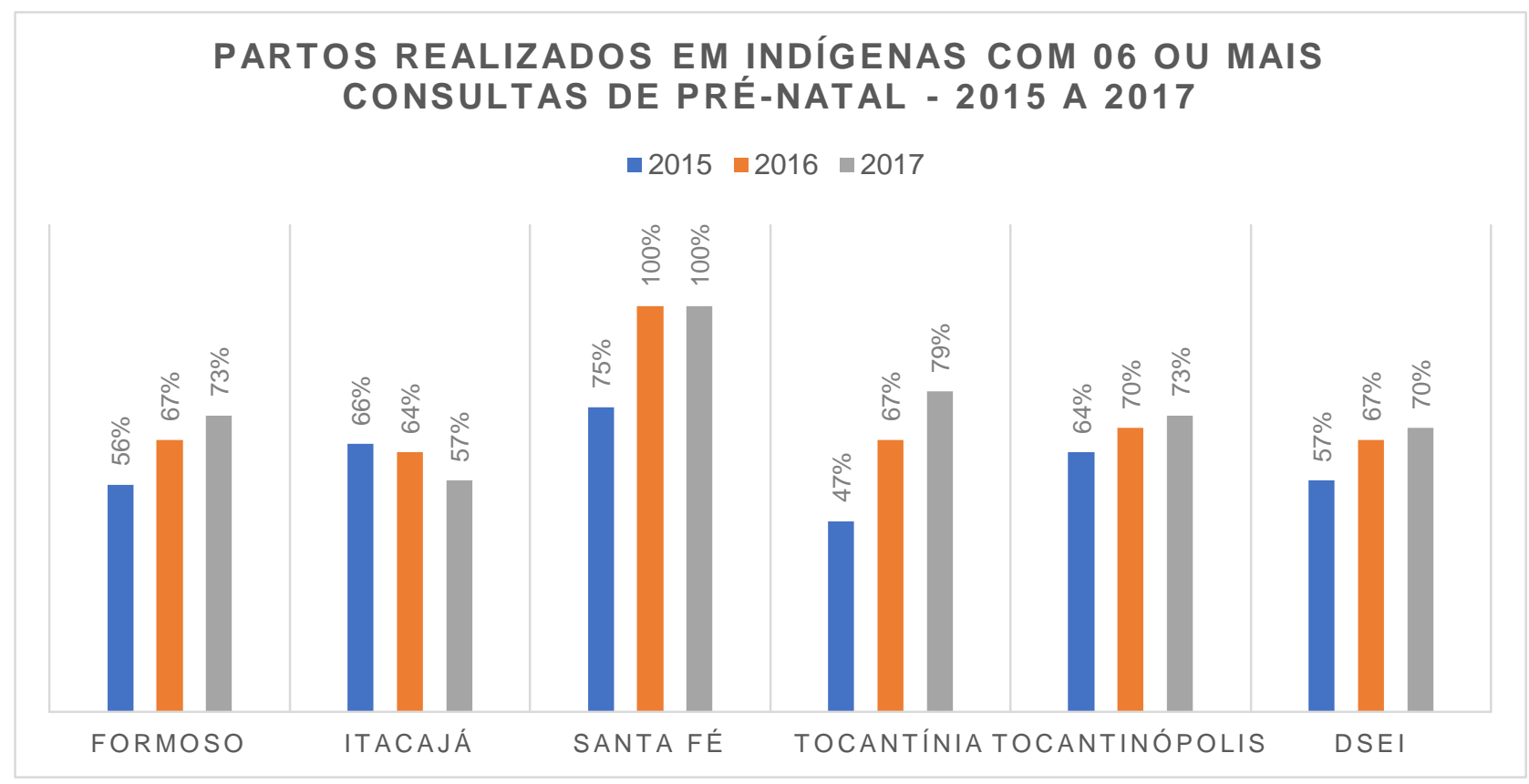

Fonte: SIASI/DSEI-TO, 2018.

O gráfico acima retrata o percentual de gestantes que pariram com 6 ou mais consultas de pré-natal nos anos de 2015 a 2017.

Analisando o gráfico acima se observa a evolução no cuidado as gestantes garantindo o quantitativo de consultas preconizadas pelo Ministério da Saúde, a incorporação do Programa Mais Médico para o Brasil (PMMB) na saúde indígena contribuiu significativamente na garantia do acompanhamento das gestantes indígenas. 
Gráfico 2: Série histórica de gestantes que pariram com exames preconizados no PréNatal - 2015 a 2017.

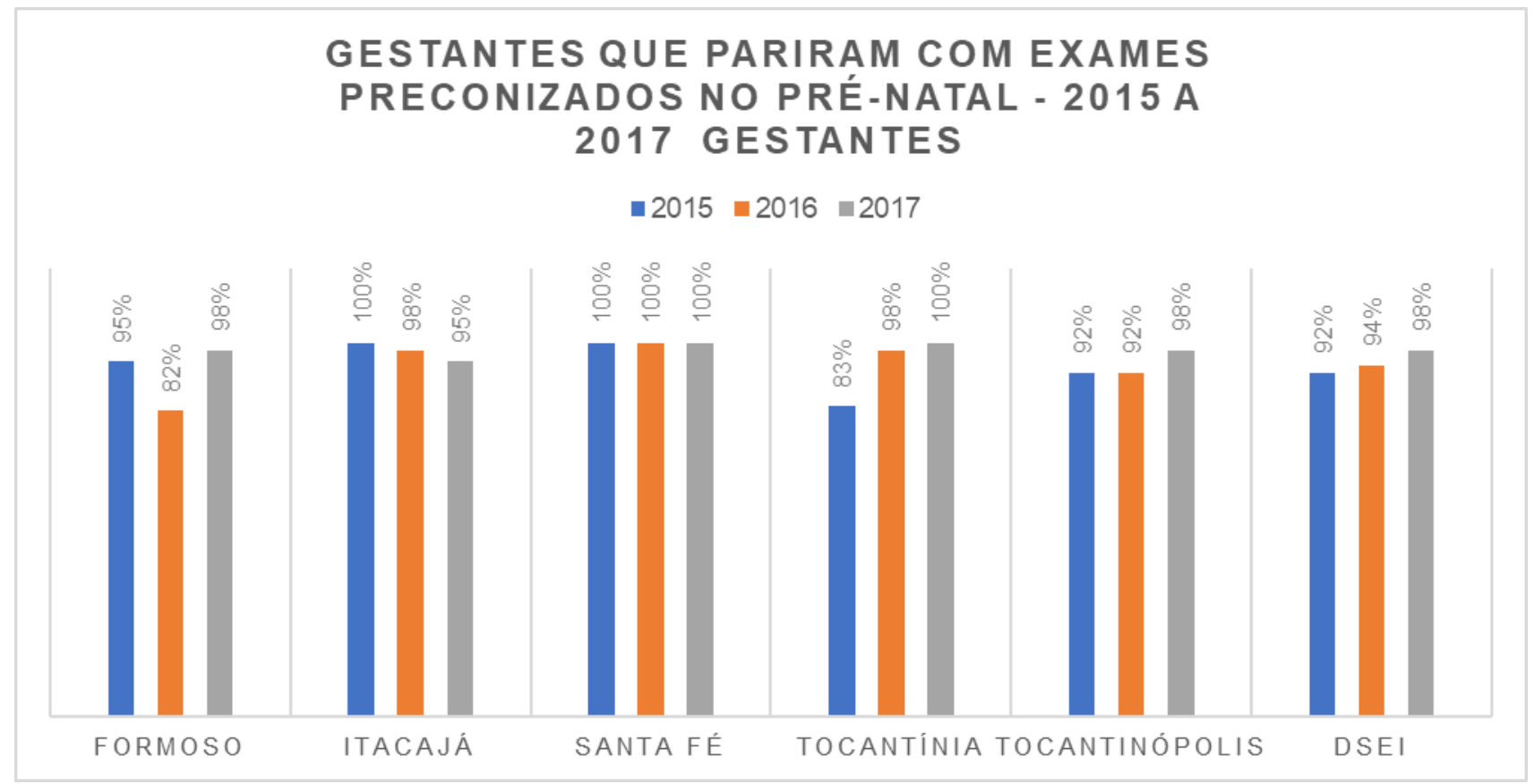

Fonte: SIASI/DSEI-TO, 2018.

O gráfico acima retrata o percentual de gestantes que pariram com os exames prénatal preconizados pelo MS: hemograma, glicemia, urina, tipagem sanguínea, VDRL (sífilis), HIV, Hepatite B por PBI, realizado nos anos de 2015 a 2017.

O gráfico abaixo descreve o atendimento odontológico no distrito de 2014 a julho de 2018, de acordo com os dados apresentados é percepetivel a progressão no número da primeira consulta odontólogia no distrito.

Gráfico 3: Série histórica 1ํㅗㄹ consulta odontológica - DSEI - TO.

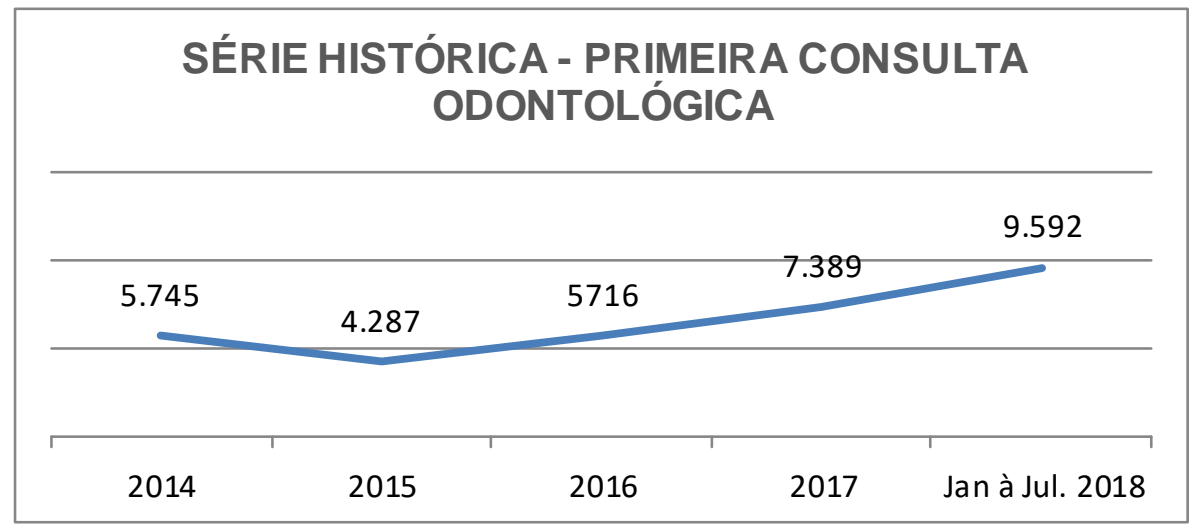

Fonte: SIASI/DSEI-TO, 2018. 
De acordo com dados do SIASI/SUS, o DSEI - TO está em primeiro lugar em relação aos 34 distritos existentes no país, com maior número de primeiras consultas realizadas no ano de 2018, conforme distribuição na tabela abaixo:

Tabela 2: Série histórica Tratamento Básico Odontológico Concluído - DSEI - TO.

\begin{tabular}{|c|c|c|c|c|c|c|c|}
\hline \multicolumn{8}{|c|}{ Tratamento Básico Odontológico Concluído - DSEI - TO } \\
\hline Faixas Etárias & $\begin{array}{l}<1 \\
\text { ano }\end{array}$ & $\begin{array}{l}1-4 \\
\text { anos }\end{array}$ & $\begin{array}{l}5-6 \\
\text { anos }\end{array}$ & $\begin{array}{l}7-59 \\
\text { anos }\end{array}$ & $\begin{array}{l}>=60 \\
\text { anos }\end{array}$ & TOTAL & MIF \\
\hline DSEI Tocantins & 342 & 1.689 & 2.031 & 492 & 3.206 & 342 & 1.689 \\
\hline $\begin{array}{l}\text { № de pessoas com } \\
\text { esquema completo }\end{array}$ & 269 & 1.559 & 1.828 & 468 & 3.035 & 269 & 1.559 \\
\hline Percentual (\%) & 78,7 & 92,3 & 90,0 & 95,1 & 94,7 & 78,7 & 92,3 \\
\hline Itacajá & 117 & 531 & 648 & 158 & 912 & 117 & 531 \\
\hline $\begin{array}{l}\text { № de pessoas com } \\
\text { esquema completo }\end{array}$ & 107 & 504 & 611 & 153 & 871 & 107 & 504 \\
\hline Percentual (\%) & 91,5 & 94,9 & 94,3 & 96,8 & 95,5 & 91,5 & 94,9 \\
\hline Formoso do Araguaia & 35 & 196 & 231 & 117 & 473 & 35 & 196 \\
\hline $\begin{array}{l}\text { № de pessoas com } \\
\text { esquema completo }\end{array}$ & 34 & 188 & 222 & 114 & 454 & 34 & 188 \\
\hline Percentual (\%) & 97,1 & 95,9 & 96,1 & 97,4 & 96,0 & 97,1 & 95,9 \\
\hline Tocantinópolis & 106 & 438 & 544 & 74 & 708 & 106 & 438 \\
\hline $\begin{array}{l}\text { № de pessoas com } \\
\text { esquema completo }\end{array}$ & 78 & 411 & 489 & 73 & 686 & 78 & 411 \\
\hline Percentual (\%) & 73,6 & 93,8 & 89,9 & 98,6 & 96,9 & 73,6 & 93,8 \\
\hline Santa Fé do Araguaia & 02 & 24 & 26 & 26 & 107 & 02 & 24 \\
\hline $\begin{array}{l}\text { № de pessoas com } \\
\text { esquema completo }\end{array}$ & 02 & 23 & 25 & 25 & 102 & 02 & 23 \\
\hline Percentual (\%) & 100,0 & 95,8 & 96,1 & 96,2 & 95,3 & 100,0 & 95,8 \\
\hline Tocantínia & 82 & 500 & 582 & 117 & 1006 & 82 & 500 \\
\hline $\begin{array}{l}\text { № de pessoas com } \\
\text { esquema completo }\end{array}$ & 48 & 433 & 481 & 103 & 922 & 48 & 433 \\
\hline Percentual (\%) & 58,5 & 86,6 & 82,6 & 88,0 & 91,7 & 58,5 & 86,6 \\
\hline
\end{tabular}

Fonte: SIASI/DSEI-TO, 2018. 
O gráfico abaixo apresenta o quantitativo de atendimentos odontológicos no distrito de 2014 a julho de 2018. Em relação ao tratamento básico concluído observa-se o avanço deste indicador, mesmo com as várias barreiras encontradas para a realização do atendimento odontólogico nas aldeias, tais como: o acesso as aldeias, as tecnólogias, a comunicação e os aspectos culturais.

Gráfico 4: Quantitativo de tratamentos odontológicos concluídos - DSEI - TO.

\section{TRATAMENTO ODONTOLÓGICO CONCLUÍDO}

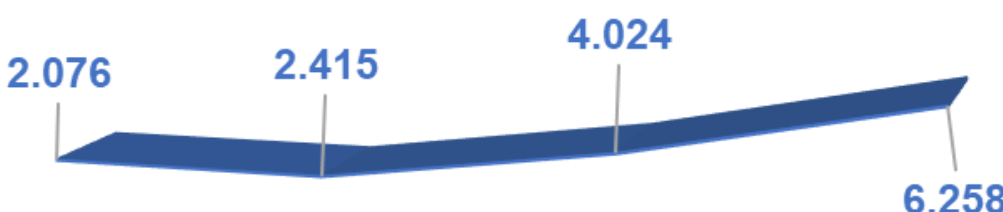

6.258

2014

2015

2016

2017

Fonte: SIASI/DSEI-TO, 2018.

Em relação ao calendário de vacinas, observa-se que houve uma pactuação com objetivo de alcançar $85 \%$ das crianças < de 5 anos com esquema vacinal completo, valor anual da meta, de acordo com o ano em curso e que o mesmo foi alcançado.

Vale ressaltar que, para a população menor de ano, ainda é um grande desafio o alcance desta meta, considerando o número de vacinas multidoses para essa faixa etária e a alta rotatividade da população entre aldeias e municípios.

Gráfico 5: Comparativo da Série Histórica da Taxa de Mortalidade Infantil entre DSEI/TO e Estado do Tocantins 2011 a 2017. 


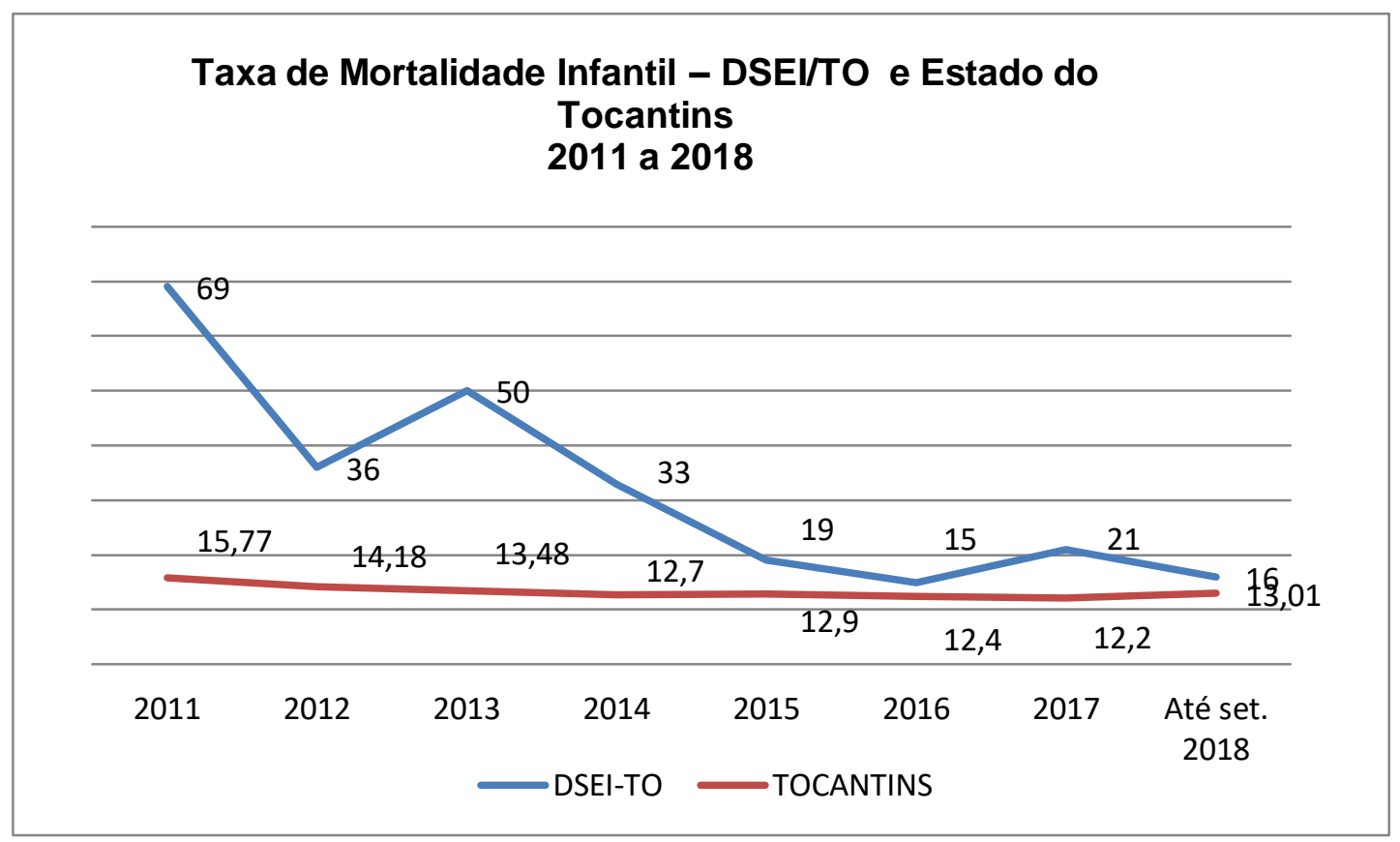

Fonte: SIASI/DSEI-TO, 2018.

Observa-se no gráfico acima que a Taxa de Mortalidade Infantil (TMI) do distrito vem apresentando uma tendência decrescente. A TMI, é um importante indicador de saúde que além de avalia as condições socioeconômicas e sanitárias de uma população, apontam o acesso e a qualidade dos recursos disponíveis para atenção à saúde materna e infantil, importante destacar os avanços apresentados pelo DSEI-Tocantins ao analisarmos a taxa de mortalidade Infantil entre 2011 a 2017 com a redução de 69/1.000 para 21/1.000 nv, este resultado pode estar relacionado como com fortalecimento da Atenção Básica no DSEI- TO, tais com aumento da força trabalho, melhoria no acesso ao serviços de MAC , garantia da realização dos exames preconizados pelo MS no pré natal e a inserção dos profissionais do Programa Mais Médicos no Brasil na Saúde Indígena.

A Atuação dos profissionais da Enfermagem no Subsistema de atenção a saúde indígena é conhecida pelas diferenças culturais e dificuldade no acesso. Então o profissional de enfermagem atuante nas áreas indígenas tem que estar, além de habilitado, ter um conhecimento específico de como atuar em cada aldeia e cada povoado indígena, assim promovendo a saúde através da prevenção e do tratamento adequado, trabalhando em equipe e seguindo o planejamento dos órgãos constituintes. (MARINELLI et al., 2012) 
De acordo João Paulo Nunes (2014), "ser Enfermeiro é ser um Profissional altamente qualificado que usa as suas competências técnicas, relacionais, éticas e culturais para o bem estar das pessoas, famílias e comunidades, é saber exercer de forma autónoma e interdependente, uma atividade insubstituível, com elevada aplicabilidade no quotidiano dos cidadãos".

O profissional de enfermagem tem como objetivo prestar cuidados ao ser humano, em todo o seu ciclo vital, e aos grupos sociais em que ele está integrado, de maneira que mantenha, melhore e/ou recupere a saúde (SOUZA et al.,2015)

Desse modo, a antropologia surge como sendo o caminho a ser percorrido para que então, possa-se oferecer uma assistência de qualidade. Assim, com base na teoria defendida por Leininger, Vilelas e Janeiro (2012) que ressaltam a importância da competência cultural como sendo imprescindível aos enfermeiros perante a pluralidade étnico cultural, aonde a competência cultura é compreendida como sendo a capacidade de respeitar, compreender as diferenças presentes em cada sociedade, a fim de prestar os cuidados de qualidade em meio a uma diversidade de indivíduos.

\section{CONCLUSAO}

Diante das evidências elucidadas no decorrer deste estudo, é possível considerar que as dificuldades enfrentadas pelos profissionais da saúde, principalmente os da enfermagem, simbolizam uma realidade que são resumidos a improvisos. Tais improvisos que podem ter sentidos opostos, ou seja: positivo, representando uma superação da profissão nas dificuldades enfrentadas, ou negativo, representando uma realidade em que a profissão sofre em colocar em prática uma atuação limitada e com incerteza da efetividade.

Porém, Silva et al. (2003) apostam que tais enfrentamentos podem representar momentos de aprendizagem que são particulares pela necessidade do "atendimento diferenciado".

Sabe-se que a assistência de enfermagem na promoção da saúde em comunidades indígenas apresenta impacto expressivo nas condições de saúde e na qualidade de vida dessa população.

Assim, faz-se necessário que haja a sensibilização dos profissionais de saúde quanto aos cuidados com os povos indígenas, visando a adoção de medidas de promoção a saúde e a prevenção de doenças. 
Vale ressaltar que a atribuição do enfermeiro é primordial, uma vez caracteriza a compreensão ampliada do processo saúde-doença que a população indígena vem sofrendo, e sua específica atuação em conjunto com tais necessidades.

O que justifica a real necessidade de trabalhos voltados para essa temática, isto a assistência vai além do cuidado, abrangendo toda a diversidade desse povo, que vive em intensa luta e conflitos pelos seus direitos e principalmente respeito.

\section{REFERENCIAS}

ALMEIDA, Severina Alves de; ALBUQUERQUE, Francisco Edviges; SILVA, Denyse Mota da; SILVA, Ângela Maria.; FERREIRA, Renato dos Reis. A Educação Escolar Intercultural Apinajé: Um Olhar para o "Professor Bilíngue". Facit Business and Technology. Journal 139, 2017; 2(1):139. Disponível em: http://revistas.faculdadefacit.edu.br/index.php/JNT/article/download/180/182. Acesso em 09 de Agosto de 2018.

ALVES, Daíse; VIEIRA, Martha Victor. Territorialidade e Demarcação de Terras Indígenas no Tocantins: O Histórico dos Karajá do Norte. Revista Nãnduty. Vol. 05, N. 7, 2017/2. Disponível em: http://ojs.ufgd.edu.br/index.php/nanduty/article/view/7798. Acesso em: 21 de Julho de 2018.

Amorim, MS. Acesso da População Indígena da Região Norte do Tocantins à Rede de Atenção à Saúde de Média e Alta Complexidade. Dissertação (Mestrado).

Universidade Federal da Bahia/Instituto de Saúde Coletiva. Salvador/BA: UFB/ISC, 2016. Disponível em:

https://repositorio.ufba.br/ri/bitstream/ri/25731/1/DISS\%20MP.\%20MARGARETH\%20AMO RIM\%202016.pdf. Acesso em: 08 de Junho de 2018.

BRASIL. Decreto no 7.336, de 19 de Outubro de 2010. Aprova a Estrutura Regimental e o Quadro Demonstrativo dos Cargos em Comissão e das Funções Gratificadas do Ministério da Saúde e dá outras providências: cria a Secretaria Especial de Saúde Indígena - SESAI dentro da estrutura do Ministério da Saúde, cabendo ao MS gerenciar diretamente a atenção à saúde dos indígenas. Antes a FUNASA era responsável pelas ações de saúde dos indígenas. Ministério da Saúde Brasília, 2010. Disponível em: http://www.planalto.gov.br/ccivil 03/ Ato2007-2010/2010/Decreto/D7336.htm. Acesso: 20 de Agosto de 2018.

Portaria MS n 852 de 30 de Setembro de 1999. Cria os Distritos Sanitários Especiais Indígenas. Ministério da Saúde, Brasília, 1999. Disponível em: http://www.planalto.gov.br/ccivil 03/ Ato2007-2010/2010/Decreto/D7336.htm. Acesso: 20 de Agosto de 2018.

Portaria MS n²54 de 31 de Janeiro de 2002. Política Nacional de Atenção à saúde dos povos indígenas. Fundação Nacional de Saúde, Brasília, 2002. Disponível em: http://bvsms.saude.gov.br/bvs/publicacoes/inca/manual_profissionaisdesaude.pdf.

Acesso: 20 de Agosto de 2018. 
Resolução COFEN n 381/201, que se refere à coleta de material para colpo citologia, Disponível em: www.portalcofen.gov.br. Acesso em: 20 de Setembro de 2018.

Portaria MS n 645 de 24 de Março de 2006, que Institui o Certificado Hospital Amigo do Índio, a ser oferecido aos estabelecimentos de saúde que fazem parte da rede do Sistema Único de Saúde (SUS). Ministério da Saúde, Fundação Nacional de Saúde, Brasília, 2006. Disponível em:

http://bvsms.saude.gov.br/bvs/publicacoes/inca/manual_profissionaisdesaude.pd. Acesso: 25 de Agosto de 2018.

Portaria MS n 1.163 de 14 de Setembro de 1999, Dispõe sobre as responsabilidades na prestação de assistência à saúde dos povos indígenas, no Ministério da Saúde e dá outras providências. Ministério da Saúde, Brasília, 1999. Disponível em: http:// bvsms. saude.gov.br/ bvs/publicacoes/inca/manual_profissionaisdesaude.pd. Acesso: 25 de Agosto de 2018.

Portaria MS n ${ }^{\circ} 1.801$ de 9 de novembro de 2015, que define os Subtipos de Estabelecimentos de Saúde Indígena e estabelece as diretrizes para elaboração de seus projetos arquitetônicos, no âmbito do Subsistema de Atenção à Saúde Indígena (SASISUS). Ministério da Saúde, Brasília, 2015.

Fundação Nacional de Saúde. Política Nacional de Atenção à Saúde dos Povos Indígenas. - 2ª edição - Brasília: Ministério da Saúde. Fundação Nacional de Saúde, 2002. $40 \mathrm{p}$

Portaria no 1.088, de 04 de Julho de 2005, Dispõe sobre a definição dos valores do incentivo financeiro de atenção básica de saúde aos povos indígenas e sobre a composição e organização das equipes multidisciplinares de atenção à saúde indígena.

NOTA TÉCNICA № 17/2018-DIASI/CGAPSI/DASI/SESAI/MS, Documento Orientador da Organização dos Processos de Trabalho da Atenção Primária à Saúde nos Distritos Sanitários Especiais Indígena. SESAI/MS.

. NOTA TÉCNICA № 018/2014-DIASI/CGAPSI/DASI/SESAI/MS, Documento Orientador das Atribuições dos Enfermeiros de Polo Base indígena no Trabalho da Atenção Primária à Saúde nos Distritos Sanitários Especiais Indígena. SESAI/MS.

Portaria MS no 475. A Secretária de Atenção à Saúde - Substitua, no uso de suas atribuições. Ministério da Saúde. Brasília-DF de 1ํ de Setembro de 2008. Disponível em: http://bvsms.saude.gov.br/bvs/saudelegis/sas/2008/prt0475_01_09_2008.html. Acesso em: 08 de Junho de 2018.

Decreto no 8.901, de 10 de Novembro de 2016. Aprova a Estrutura Regimental e o Quadro Demonstrativo dos Cargos em Comissão e das Funções de Confiança do Ministério da Saúde. Brasília, 2016. Disponível em: http://bvsms.saude.gov.br/bvs/publicacoes/regimento_interno_ministerio_saude.pdf. Acesso: 29 de junho de 2018. 
BRASIL. Ministério da Saúde. Secretaria de Gestão do Trabalho e da Educação na Saúde. Departamento de Gestão da Educação na Saúde. Programa de Qualificação de Agentes Indígenas de Saúde (AIS) e Agentes Indígenas de Saneamento (AISAN) / Brasília: Ministério da Saúde, 2016. Disponível em:

http://bvsms.saude.gov.br/bvs/publicacoes/saude_jovem_adulto_idoso_volume_1.pdf . Acesso em: 07 de Setembro de 2018.

BRASIL. Ministério da Saúde. 5a Conferência Nacional de Saúde Indígena: Relatório Final/ Ministério da Saúde, Conselho Nacional de Saúde - Brasília: Ministério da Saúde, 2015. 305 páginas.

COFEN. Conselho Federal de Enfermagem. Resolução 311/2007 - aprova a reformulação do código de Ética dos Profissionais de Enfermagem. Disponível em: www.portalcofen.gov.br. Acesso em: 17 de outubro. 2018.

DSEI/TO. Distrito Sanitário Especial Indígena do Tocantins. Cartografia/2017. Secretaria Especial de Saúde Indígena. Ministério da Saúde.

GUSMAN, Christine Ranier. Parteiras Indígenas e os Objetos do Partejar: Apropriação, usos, sentidos e significados. Tese Doutorado. Universidade Federal de São Paulo. Escola Paulista de Medicina. Programa de Pós-Graduação. São Paulo 2017. Disponível em: http://repositorio.unifesp.br/bitstream/handle/11600/41854/20170010.pdf;jsessionid=0AEE36F44C694AFA1027D7B60EC4C49E?sequence $=1$. Acesso em 14 de Junho de 2018.

LANGDON, EJ. CARDOSO, MD. Saúde Indígena: Políticas comparadas na América Latina/ Organizadoras, - Florianópolis: Ed. da UFSC, 2015. Disponível em: http://www.scielo.br/scielo.php?script=sci_arttext\&pid=S0102-311X2017000308001. Acesso em: 23 de Julho de 2018.

LIMA, Layanna Giordana Bernardo. Os Akwê-Xerente do Tocantins: território indígena e as questões socioambientais. Tese de Doutorado. Faculdade de Filosofia, Letras e Ciências Humanas da Universidade de São Paulo. São Paulo 2016. Disponível em: www.teses.usp.br/teses/disponiveis/.../2017_LayannaGiordanaBernardoLima_VCorr.pdf. Acesso em 18 de Outubro de 2018.

MARTINS, André Luiz. Política de saúde indígena no Brasil: reflexões sobre o processo de implementação do Subsistema de Atenção à Saúde Indígena. Dissertação de Mestrado. Escola Nacional de Saúde Pública Sergio Auroca, Rio de Janeiro, 2013. Disponível em: https://bvssp.icict.fiocruz.br/lildbi/docsonline/get.php?id=3822. Acesso em: 26 de Setembro de 2018.

Martins MJR, Fernandes SJD. A Visibilidade Da Enfermagem, Dando Voz À Profissão: Revisão Integrativa. J. Nurse UFPE on line , 2014. Disponivel em http://www.revista.ufpe.br/revistaenfermagem/index.php/revista/article/view/6219/pdf 5713 . Acesso em: 19 de Outubro de 2018. 
Indígena. Dissertação de Mestrado. Universidade Federal do Tocantins, Araguaína Tocantins, 2017. Disponível em:

http://uft.edu.br/lali/uploads/dissertac\%CC\%A7a\%CC\%83osimara.pdf. Acesso em: 30 de Abril de 2019.

NUNES, J.P. O que é ser Enfermeiro? Escola Superior de Enfermagem S. Francisco das Misericórdias, 2014. Disponível em: <http://www.enfermagem.edu.pt/Informacoes/o-que-eser-enfermeiro.html >. Acesso em: 19 de outubro de 2018.

PARADA, R. et al. A política nacional de atenção oncológica e o papel da atenção básica na prevenção e controle do câncer. Rev. APS, v.11, n. 2, p.199 - 206, abr./jun. 2008.

PONTES, Ana Lucia de Moura. Atenção diferenciada e o trabalho do agente indígena de saúde na implementação da Política de Saúde Indígena. 2013. Tese de Doutorado. Escola Nacional de Saúde Pública Sérgio Arouca. Fundação Oswaldo Cruz.

Portal Saúde - SUS. Disponível em

:<http://bvsms.saude.gov.br/bvs/publicacoes/inca/manual_profissionaisdesaude.pdf

Acesso em: 17 de Outubro de 2018.

RIBEIRO, Darcy. 1979. Os índios e a civilização: a integração das populações indígenas no Brasil moderno. 3ª edição. Petrópolis, Vozes.

RUTHES. RM, CUNHA. ICKO. Competências do enfermeiro na gestão do conhecimento e capital intelectual. Rev Bras Enferm (internert). 2009. Disponível em http://www.scielo.br/pdf/reben/v62n6/a16v62n6.pdf. Acesso em: 17 de Outubro de 2018.

SILVA, Nair Chase da; GONCALVES, Maria Jacirema Ferreira; LOPES NETO, David. Enfermagem em saúde indígena: aplicando as diretrizes curriculares. Rev. bras. enferm. [online]. 2003, vol.56, n.4, pp.388-391. ISSN 0034-7167. Disponível em: http://dx.doi.org/10.1590/S0034-71672003000400016. Acesso em: 17 de Outubro de 2018.

SOUZA, Kariny Teixeira de. O Faccionalismo Krahô-Kanela à Luz da Teoria da Dádiva de Marcel Mauss. Revista de Ciências Humanas e Sociais da FSDB - Ano V, Vol. IX. Janeiro a Junho de 2009. Disponível em: https://www.fsdb.edu.br/fsdb/wpcontent/uploads/2018/06/Ethos-Epsiteme-9-Eletronica.pdf. Acesso em: 02 de Setembro de 2018. 